\title{
Performance of Carrier- Suppressed Return-to-Zero(CSRZ), Duo binary Return-to-Zero(DRZ) and Modified Duo binary Return-to-Zero(MDRZ) Modulation Methods in WDM-PON Systems
}

\author{
Yankho Alexander Chitungu, Gurmeet Singh, Dinesh Kumar Verma
}

\begin{abstract}
In this paper the different modulation techniques used in fiber optic technology are compared to check which one has the better bit error rate (BER). There are a number of different modulation techniques used in WDN-PON systems but here the comparison is made among carrier-suppressed return-to-zero (CSRZ), duobinary return-to-zero (DRZ) and modified duobinary return-to-zero (MDRZ) modulation. The comparison to determine which is better is made my performing simulations in OptiSystem version 17 software. OptiSystem 17 is the current version of the software at the time the simulations were being carried out. In the OptiSystem software an optical fiber system is simulated from transmitter all the way to receiver. In the experiment when checking the performance of the different modulation formats, the optical fiber and the receiver are left unchanged and only the transmitter part is changed according to the modulation format being simulated at that particular instance. The modulation in the transmitter is done by using proper components from the OptiSystem library which when organized in the right order will give out a signal modulated in a certain format by the time it is fed into the optical fiber. Knowing the performance of modulation can help in WDM PON where the network gets to use passive components as it goes to user. Since no active amplification or correction equipment is used in WDM-PON, therefore using the format with the best performance can help with performance of overall system.
\end{abstract}

Keywords: Bit-error-rate, WDN-PON, Carrier suppressed return to zero, duobinary return to zero, modified duobinary return to zero.

\section{INTRODUCTION}

The internet has become a basic necessity for our day to

Revised Manuscript Received on July 02, 2020.

* Correspondence Author

Yankho Alexander Chitungu, Department of Electronics and Communication Engineering, PDM University, Bahadurgarh, India. E-mail: ychitungu@gmail.com

Gurmeet Singh, Assistant Professor, Department of Electronics and Communication Engineering, PDM University, Bahadurgarh, India. E-mail: gurmeet_engg@pdm.ac.in

Dinesh Kumar Verma, Assistant Professor, Department of Electronics and Communication Engineering, PDM University, Bahadurgarh, India. E-mail: dinesh3_engg@pdm.ac.in

(C) The Authors. Published by Blue Eyes Intelligence Engineering and Sciences Publication (BEIESP). This is an open access article under the CC BY-NC-ND license (http://creativecommons.org/licenses/by-nc-nd/4.0/) day life and with the growth is use of smartphones and online video streaming services like Netflix and Disney+ the requirement of high band width has become a must. Fiber optic use has grown rapidly in the past decade as the world has become more connected than ever before, this has allowed increased data rates which were hard to achieve with old electronics media.

\section{LITERATURE REVIEW}

\section{A. Passive optical network}

Passive Optical Network (PON) which is also referred to as a FTTH (Fiber to the Home) is a technology which has been deployed for use in both domestic and commercial consumers. Passive Optical Network uses a point to multipoint topology to connect end users to the service provider. This technology uses passive splitters which do not require any power source to operate. This is advantageous as it helps reduce the cost by avoiding expensive optic-electronic conversions. Fig 2.1 is an example of a PON.

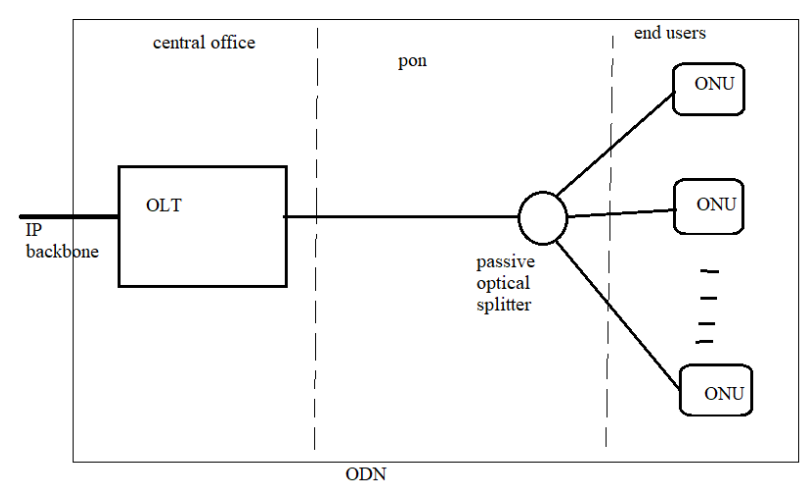

Fig 1 passive optical network

The IP backbone is a group if high speed transmission paths usually made up of fiber optic cable and the provide networking facilities to different broadband service providers from around the world.

Published By:

Blue Eyes Intelligence Engineering and Sciences Publication

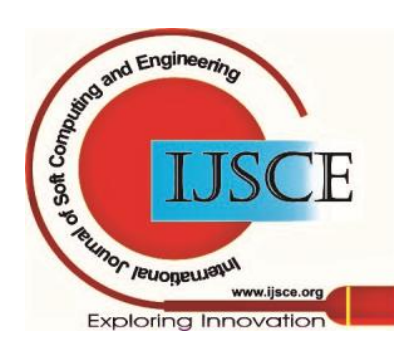


These are called optical distribution networks (ODN) and are made up of components like fiber cable, and splitters, there is no powered equipment required as traditionally it's all passive. The optical distribution network includes the OLT and ONU.

Optical line terminal (OLT) is the network element that controls the downstream upstream signals, this is done by combining the signals together when they are upstream and sharing the signal to each end user unit that is sharing a fiber cable. The signals are combined using different multiple access protocols for example time division multiple access (TDMA) or wavelength division multiple access protocol (WDMA). Here encryption is used to make the transmission secure. The optical network unit (ONU) is located at the end user premises, this is where the optical connection is terminated at the user side.

\section{B. Wavelength Division Multiplexing Passive Optical Network}

WDM optical fiber systems use a laser which can transmit multiple wavelength into fiber at same time. Take for example the fiber can have 300000 channels each of which can use $10 \mathrm{Mhz}$, advances in the laser industry have made it possible to put multiple channels into a single fiber optic cable. When the channels are closely packed then it's usually called dense wavelength division multiplexing (DWDM). To avoid unnecessary interference the ITU said the wavelengths have to be on a fixed grade known as the ITU grade which uses a standard called ITU G.692. This ITU G.692 standard uses the reference frequency of $193.1 \mathrm{Thz}$ which is on the $1552 \mathrm{~nm}$. The spacing used is $100 \mathrm{Ghz}$ which is approximately $0.8 \mathrm{~nm}$. The WDM PON architecture is shown in the fig 2.2 above, multiple transmitters each operating in a dedicated wavelength are multiplexed together and sent into the optical fiber. When it reaches the other side, the signal is demultiplexed into the individual wavelengths and sent to specific end users fiber is there to solve the problem of the step index fiber, it does this by having a core with varying density which make the modes which travel further to also move faster than the modes which move closer to the core resulting into an almost uniform speed of transmission of all the modes. Nowadays graded index fiber is the more commonly used compare to the step index fiber because of the reduced modal dispersion and also due to the fact that the graded index has a bandwidth which is higher when compared to the step index.

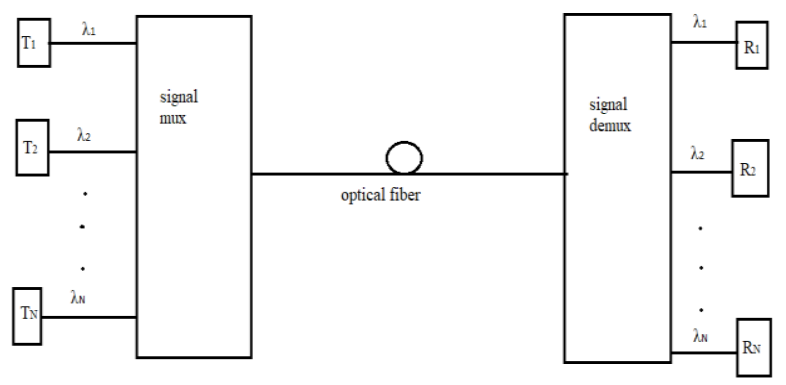

Fig 2 wavelength division multiplexing

\section{SIMULATIONS}

In the experiment three different modulation techniques are investigated to see the one which has the better bit error rate (BER) and better $\mathrm{Q}$ factor. All the three modulation techniques will use the same transmission and receiver setup to make sure that the results are only affected my change at the transmitter side.

The Loop control is used to allow for system performance calculation which is based on the amount of fibers and EDFA spans connected, with the loop control for the experiment the number of times the signal can propagate in the connected components has been set to 6 times.

The transmission path is composed of single mode fiber (SMF), erbium doped fiber amplifier (EDFA) and dispersion compensating fiber (DCF). The SMF length is set to $25 \mathrm{~km}$ and after that it connected to an EDFA with gain of $5 \mathrm{~dB}$ and noise figure of $6 \mathrm{~dB}$, then a DCF of length $5 \mathrm{~km}$ is connected after which an EDFA of gain $5 \mathrm{~dB}$ and noise figure of $6 \mathrm{~dB}$ is connected thereafter it is fed back into the loop control.

The output of the loop control is connected to a photodetector which is then connected to a low pass Bessel filter set at cutoff $0.8 *$ bit rate.

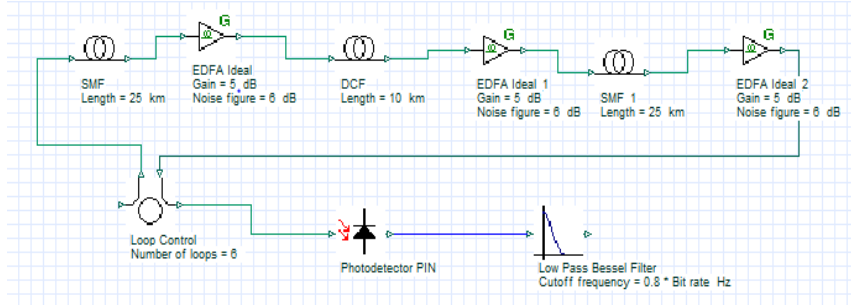

Fig 3 transmission path and receiving end of Simulated network

\section{A. Carrier suppressed return to zero}

For the transmitter component of the CSRZ a pseudo random bit sequence generator has been used to create the initial signal which is the fed to the NRZ pulse generator. The $\mathrm{CW}$ (continuous wave) laser has power set to $4 \mathrm{~mW}$ and the frequency of 193.1 which is according to the ITU G.692 standard, both the output of the NRZ pulse generator and the CW laser are connected to the first LiNb Mach-Zehnder Modulator. The output of the first LiNb Mach-Zehnder Modulator together with the output from the sine generator are fed into the second LiNb Mach-Zehnder Modulator to give the CSRZ signal which is to be transmitted over the optical fiber. The Sine Generator frequency is set to $20 \mathrm{GHZ}$ and the phase is set to -5 degree.

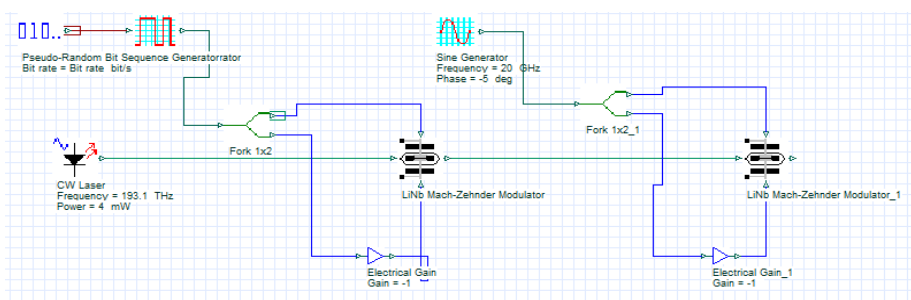

Fig 4 CSRZ modulation circuit

Published By:

Blue Eyes Intelligence Engineering and Sciences Publication (C) Convriaht: All riahts reserved. 


\section{B. Duobinary return to zero}

The Duobinary transmitter has a pseudo random bit sequence generator whose output is split into two, one is fed into the RZ pulse generator and the other is connected to a binary NOT which is then connected to a precoder with delay set to 1 , the output of the precoder is again split into two using a 1x2 fork. First link is fed to the NRZ Pulse Generator the output of which is fed to the Duobinary Pulse Generator together with other output from the precoder. The output of the Duobinary Pulse Generator together with the output of the CW laser are fed into the first LiNb Mach-Zehnder Modulator, the laser has frequency set to $1552.52438115 \mathrm{~nm}$ and power set to $4 \mathrm{~mW}$. Then the output of the first LiNb Mach-Zehnder Modulator and the output from the Sine Generator are connected into the second LiNb Mach-Zehnder Modulator whose output is the signal being transmitted on the optical fiber, the Sine Generator frequency is set to $40 \mathrm{Ghz}$ and phase is -95degree.

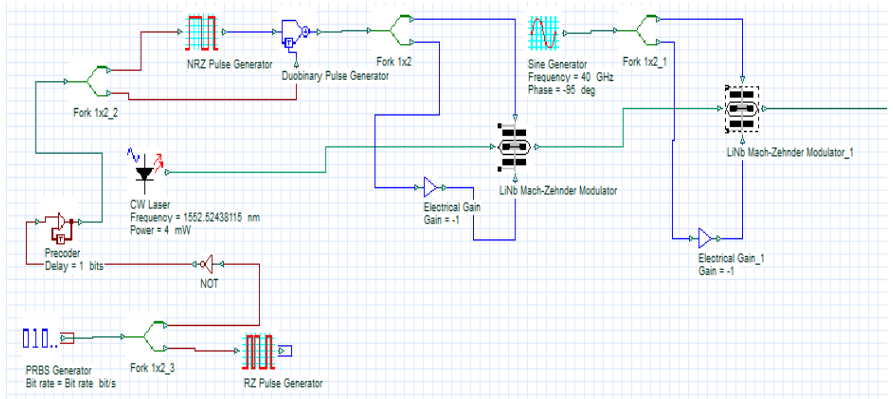

Fig 5 DRZ modulation circuit

\section{Modified duobinary return to zero}

Here we have a pseudo random bit sequence generator whose output is split into RZ Pulse Generator and the other is connected to the Duobinary precoder with delay set to 1 . The output of the Duobinary Precoder is connected to NRZ Pulse Generator which is then connected to a $1 \times 2$ fork. One output of the fork is connected to the Electrical Time Delay with delay set to 1/Bit rate then to the Electrical Subtractor and the other fork output is connected directly to the Electrical Substractor. the output of the Electrical Substractor is connected to the LiNb Mach-Zehnder Modulator with the CW laser having a frequency of $193.1 \mathrm{Thz}$ and power of $4 \mathrm{mw}$. The output of this first LiNb Mach-Zehnder Modulator is connected to the input of the second LiNb Mach-Zehnder Modulator with the output from the Sine Generator which has a frequency set to $60 \mathrm{Ghz}$ and phase set to -90 degree.

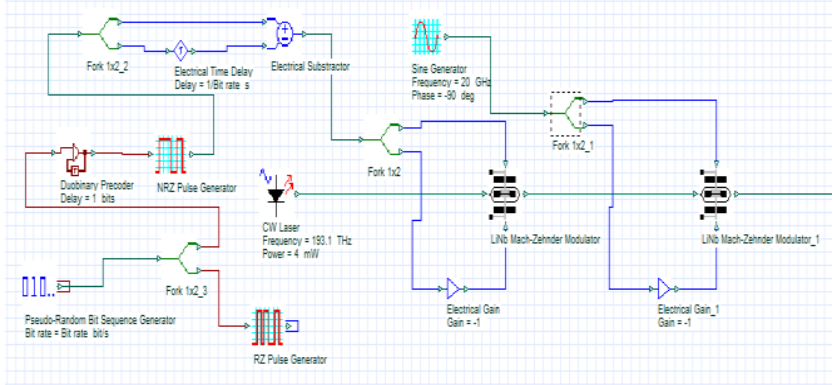

Fig 6 MDRZ modulation circuit

\section{RESULTS}

The eye diagram is used to show the quality of the received signal, the more open the eye diagram, the better the signal to noise ratio and if the eye diagram is not open the signal to noise ratio is poor. Bit error rate in is the ratio of transmitted signal to the total detected errors in the bits at the receiver, the lower the error rate the better performing the system. After running the simulation in OptiSystem 17 and connecting the circuits as shown then the following eye diagrams were obtained for each advanced modulation technique simulated

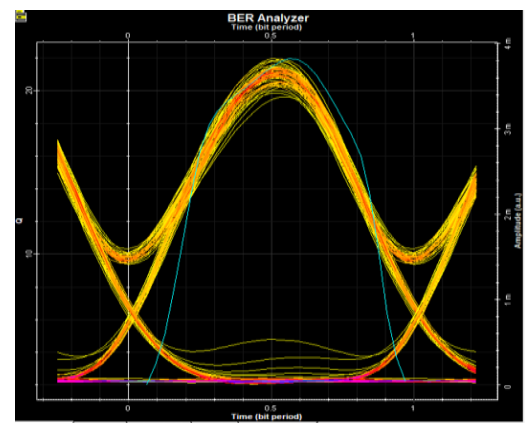

Fig 7 CSRZ eye diagram

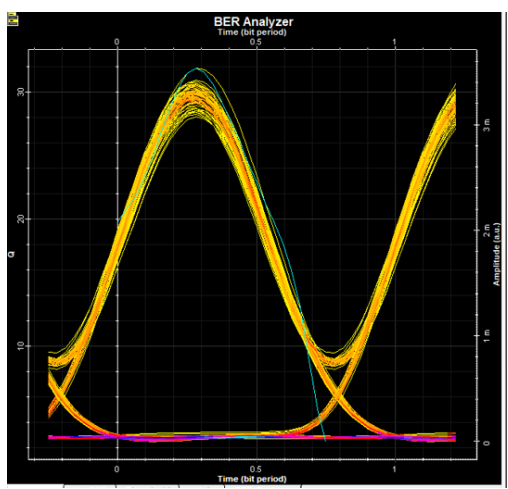

Fig 8 DRZ eye diagram

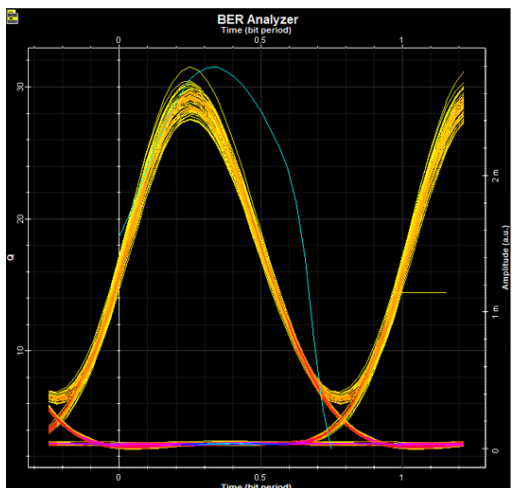

Fig 9 MDRZ eye diagram

Table 1 Results obtained for the CSRZ, DRZ, MDRZ modulation techniques

\begin{tabular}{|l|l|l|l|}
\hline & \multicolumn{1}{|c|}{ CSRZ } & \multicolumn{1}{c|}{ DRZ } & \multicolumn{1}{c|}{ MDRZ } \\
\hline Max. Q factor & 21.9542 & 31.8546 & 31.4786 \\
\hline Min. BER & $3.90717 \mathrm{e}-107$ & $3.91854 \mathrm{e}-223$ & $5.42657 \mathrm{e}-218$ \\
\hline
\end{tabular}

Published By:

Blue Eyes Intelligence Engineering and Sciences Publication 
Performance of Carrier- Suppressed Return-to-Zero(CSRZ), Duo binary Return-to-Zero(DRZ) and Modified Duo binary Return-to-Zero(MDRZ) Modulation Methods in WDM-PON Systems

\begin{tabular}{|l|l|l|l|}
\hline & \multicolumn{1}{|c|}{ CSRZ } & \multicolumn{1}{c|}{ DRZ } & \multicolumn{1}{c|}{ MDRZ } \\
\hline Eye Height & 0.00308464 & 0.00292991 & 0.00227248 \\
\hline Threshhold & 0.00163258 & 0.00041884 & 0.000219859 \\
\hline Decision inst. & 0.5625 & 0.28125 & 0.34375 \\
\hline
\end{tabular}

ECE from the university of Malawi in 2017 and is currently pursuing his M.tech in PDM university. After completing his undergraduate degree, he worked as an intern at ImoSyS systems which is a company that provides innovative solution to different companies and organizations in Malawi. He has worked on different projects including being part of a team which designed and deployed a TB cases tracking and monitoring system for the government of Malawi in collaboration with the world bank while at ImoSyS. He has been part of the team which designed and deployed a water management system for Lilongwe water board in Malawi also while at ImoSyS.

Email: ychitungu@gmail.com

\section{CONCLUSION}

From the above experiment it is possible to conclude confidently that for the different types of advanced modulation techniques which are CSRZ, DRZ and MDRZ that duobinary is better than the other two methods as it has the highest value of max. Q factor and lowest value of min. BER. CSRZ is the worst of the three methods since it has the lowest max Q factor of 21.9542 and highest min. BER of 3.90717e-107. From the results it is also observed that the performance of MDRZ modulation is not far from the performance of DRZ. Therefore, for use in WDM-PON system, DRZ has the best performance as shown by the results obtained.

\section{REFERENCES}

1. P. Dane and H. Kaushal, "Characterization of RoF GPON performance for different modulation schemes," 2013 Tenth International Conference on Wireless and Optical Communications Networks (WOCN), Bhopal, 2013, pp. 1-6, doi: 10.1109/WOCN.2013.6616211.

2. S. Garg, M. Aggarwal and A. Dixit, "Power, Cost and Reach Based Evaluation of Next Generation Passive Optical Networks Architectures," 2018 IEEE International Conference on Advanced Networks and Telecommunications Systems (ANTS), Indore, India, 2018, pp. 1-6, doi: 10.1109/ANTS.2018.8710127.

3. Saleh, Zeyad. (2016). design study and simulation of a digital fiber communication system using (optisystem.10). 10.5281/zenodo.60837.

4. Rokkas, Theodoros \& Katsianis, Dimitris \& Kamalakis, Thomas \& Varoutas, Dimitris. (2011). Economics of Time and Wavelength Domain Multiplexed Passive Optical Networks. Optical Communications and Networking, IEEE/OSA Journal of. 2. 1042 1051. 10.1364/JOCN.2.001042.

5. Kavuta, Karisha. (2018). Performance of Fiber Optic Modulation Scheme on Fiber-To-The-Home Networks. International Journal of Scientific and Engineering Research. 9. 1868-1874.

6. Alvarez Guerrero, Jesús \& Amaya-Fernández, Ferney. (2017). Comparison of electronic compensation techniques in access networks with optical phase modulation and coherent detection. Revista Ingeniería y Competitividad. 19. 87-96.

7. Sri Abirami Devi.S1, Dr.N.Victor Jaya(2018) performance analysis of optical transmission system using different modulation format. International Journal of Advanced Research in Science and Engineering. Volume No.07, Special Issue No. (02), March 2018. 2319-8354

8. Ritchie, S. (1991). Fiber optic communications design handbook: Robert J. Hoss Prentice-Hall International, 1990, ISBN 0-1331-3321-4, pp xii + 435 .

9. Khadir, A.A., Dhahir, B.F., \& Fu, X. (2014). “Achieving Optical Fiber Communication Experiments by OptiSystem”. , International Journa of Computer Science and Mobile Computing, Vol.3 Issue.6, June2014, pg. 42-53

10. Y. Miyamoto(2020). Advanced Modulation Formats [online]. Available: https://optiwave.com/category/resources/

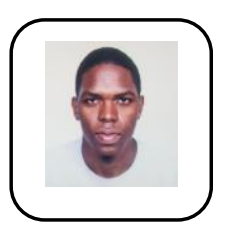

\section{AUTHORS PROFILE}

Yankho Alexander Chitungu M.tech student in Electronics and Communication Engineering Department with the PDM University, Bahadurgarh, India . He received his honors bachelor's degree in

Retrieval Number: A34520710120/2020@BEIESP

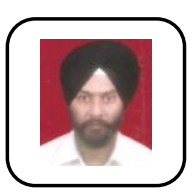

Gurmeet Singh Assistant Professor in Electronics and Communication Engineering Department with the PDM University, Bahadurgarh, India. He has received his B.E. degree in ECE from I.E.T.E. in 2005 and M.Tech degree in Signal Processing from M. D. University, Rohtak in 2009. He has more than 11 years of vast experience in teaching and other 3.5 years of experience in the field of electronics and communication engineering and more than 9 Years in teaching as an Assistant Professor in ECE in PDM College of Engineering now PDM University. He is the life time member of I.E.T.E. He has 8 Research papers in National and International Journals and Conferences. He has also guided ten (10) M.Tech. students for their research work and also many students for their Project work at UG and PG level. His research interest includes Signal Processing, Control System, Communication Systems.

Email: gurmeet_engg@pdm.ac.in

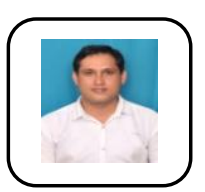

Dinesh Kumar Verma Assistant Professor in Electronics and Communication Engineering Department with the PDM University, Bahadurgarh, India. He has received his B.E. degree in ECE from M.D. University, Rohtak in 2004, M.Tech degree in Electronics and Communication Engineering from M.D. University, Rohtak in 2011 and $\mathrm{PhD}$ (pursuing) dcrust Murthal from AUG 2018. He has more than 10 years of vast experience which includes 3.2 years in reputed industry in networking like Time Broadband Services Pvt. Ltd., Tata Communication Ltd. (Formerly known as VSNL), CMS Computers Ltd. and more than 5 Years in teaching as an Assistant Professor in ECE in PDM College of Engineering. He is also CCNA Certified and also working here as a Certified CCNA trainer authorized by Cisco Networking Academy i.e. www.netacad.com which is a global program run by CISCO System directly. He is life member of ISTE Society. He has 12 Research papers in National and International Journals and Conferences. He has also guided ten (10) M.Tech. students for their research work and also many students for their Project work at UG and PG level. His research interest includes Very Large-Scale Integration (VLSI), Wireless Communication and Networking. Email: dinesh3 engg@pdm.ac.in
Published By:

Blue Eyes Intelligence Engineering and Sciences Publication

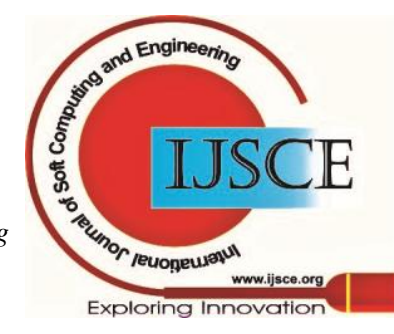

